\title{
Ecotourism Potential Assessment of Dhera Dilfekar Block, Arsi Mountains National Park, Eastern Central Rift valley of Ethiopia
}

\author{
Ziyad Jemal $^{1^{*}} \quad$ Kedir Erbo $^{1} \quad$ Abdi Haji ${ }^{2}$ \\ 1.Department of Natural Resource Management, College of Agriculture and Environmental Science, Arsi \\ University, Asella, Ethiopia \\ 2.Department of GIS and Remote Sensing, Wondo genet College of Forestry and Natural Resources, Hawassa \\ University
}

\begin{abstract}
Ethiopia holds substantial ecotourism potentials that have to be promoted for economic development. This study was done with the objective of assessing ecotourism potentials of Dhera dilfekar block for ecotourism development. Dhera dilfekar block is one of the four blocks of Arsi Mountains National Park. Seven (7) major tourist attraction sites were identified namely; Dire kiltu hyena valley, Dire kiltu lesser kudu site, Dire kiltu greater kudu site , Fachiso greater kudu site , Gara dima hyena valley(1), Awash bishola dhagaa taa'aa valley and Gara dima hyena valley(2).Additionally, the park can offer the visitor an opportunity to see lowland game species, a variety of bird species, Acacia dominated plant species with spectacular landscape, historical and Cultural sites which make the parks unique features to attract eco tourists. The block is the nearest to Addis Ababa (Finfinne) from any other Parks of our country and is found on the main asphalt road which goes to Bale Zone via Adama-Asella. Dhera dilfekar block is found in the lowland that situated in altitudinal elevation ranges from 1300 masl to 1835 masl. This makes the block to be visited all over the year for both domestic and international tourists since there is no mud to travel by vehicle and on foot. The proximity to Sodere resort hotel also brings additional opportunity to be visited by both domestic and foreign tourists. It can be concluded that the area is endowed with high Ecotourism potentials. It can also be good potential for bird watching tourism that can integrate economic gain with biodiversity conservation. Hence, urgent conservation measures and further detail research is recommended to promote this Ecotourism potentials.
\end{abstract}

Key words:Ecotourism, Arsi Mountains, Potential, Dhera dilfekar, Attraction

DOI: $10.7176 / \mathrm{JTHS} / 55-01$

Publication date:March $31^{\text {st }} 2021$

\section{INTRODUCTION}

The tourism industry is considered one of the largest and fastest growing industries in the world (Cooper \& Hall, 2008).The growing dynamics and increased destinations turned tourism to become a driver for socio-economic progress and the main income sources for many developing countries in which Ethiopia is apart (Papatheodorou, 2006). Ethiopia is known as a land of origins and biodiversity, a country of several ethnic groups, and a symbol of freedom who retained her sovereignty even during the colonial era has abundant natural and historical tourist attractions areas (Sinha, 2001).However, the tourism industry has not tapped its full potential to attract tourists in large numbers owing to poor investment in infrastructure development and inadequate promotion (UNWTC, 2010). The economy of Ethiopia has prospered for many years on agricultural products but currently, the country expands to industrialization and service providing for additional incomes through Ecotourism industry (Alamneh Amare, 2015). Proper understanding of the nature, significance and importance of these resources are essential for decision makers at all levels to make appropriate plans for improving the ecotourism in the regions (Mustefa Sultan and Teyiba Amano, 2017). However, the Eco tourism and conservation practices are still at low attention in the study area. Therefore, the aim of this study was to assess and pointed out the attractive natural and cultural resources having Ecotourism potential to be developed in Dhera Dilfekar block of Arsi Mountains National Park.

\section{MATERIALS AND METHODS}

\subsection{Description of the Study Area}

2.1.1. Location: The study was conducted in Dhera Dilfekar block, Arsi Mountains National Park, Eastern central rift valley of Ethiopia. Dhera Dilfekar block is located in Dodota district in the North eastern part of Arsi zone, Oromia Regional State. The geographic location of the study area is between $8^{0} 19^{\prime}-8^{0} 22^{\prime} \mathrm{N}$ latitude and $39^{\circ} 19^{\prime}-3922^{\prime}$ E longitude (Figure 1). The block was Adjacent to Dhera town, $25 \mathrm{~km}$ south from Adama town and $125 \mathrm{~km}$ far apart from Addis Ababa (Finfinne) town. It's also $15 \mathrm{~km}$ from the Sodere resort center in the south west, which is the most popular resort area, frequently visited. 


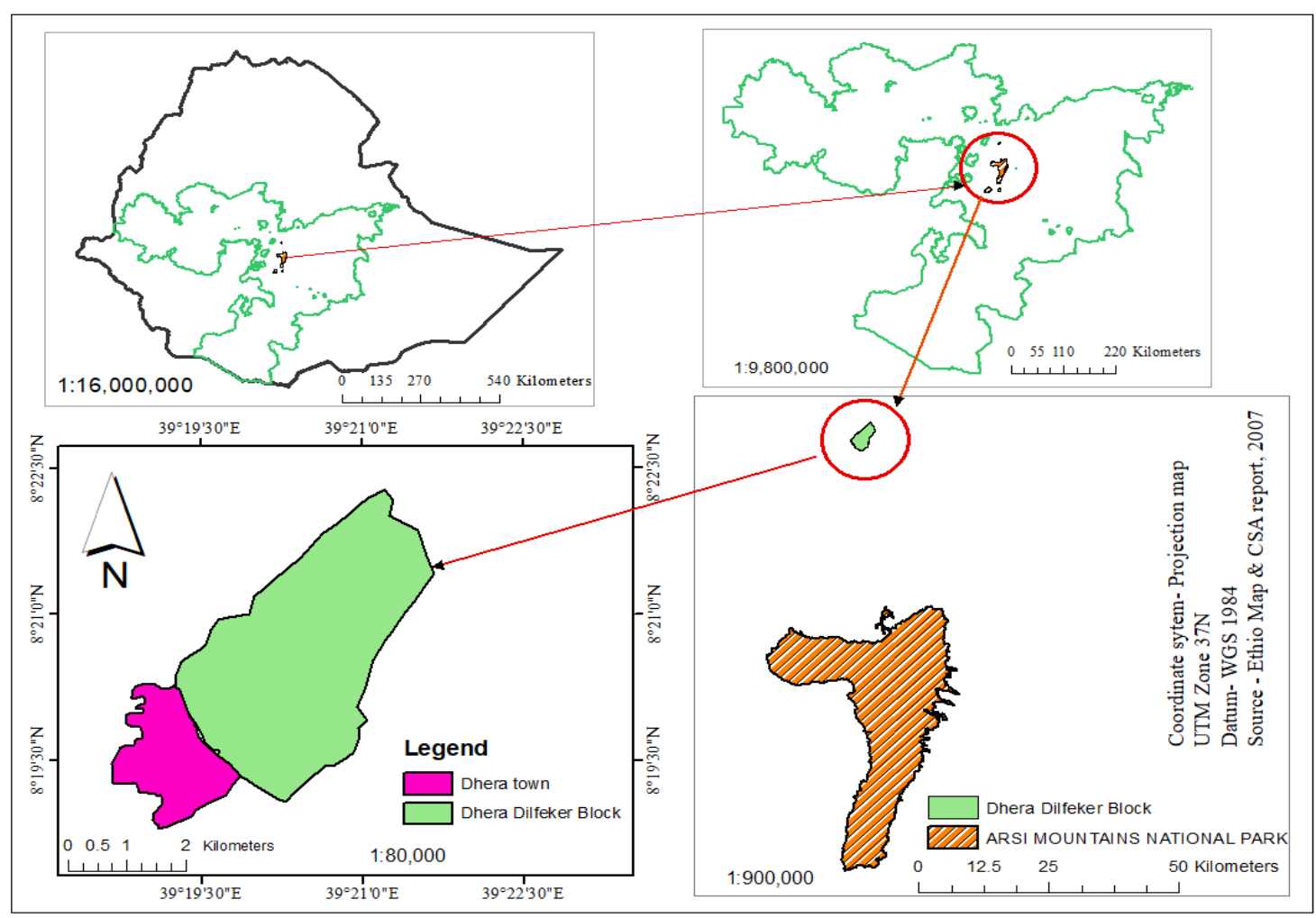

Figure 1. Map of the Study Area

According to local elders evidences the term 'Dhera' is an Afan Oromo word which sense 'long height'. This 'long height' implies to hero person called 'Shek zulfekar' who lived in this area for many years. Since he was too long and a place was called simply Dhera-Dilfekar. This conservation block renamed after this famous person lived in the area.

The total area of block was 1341 hectares. This block is bordered with five kebeles; Dhera town [kebele 01 $\&$ 02] to the south west, Awash-Bishola to the north, Dilfekar to the south east and Dire-Kiltu to the east. The block is found in the edge of eastern central rift valley parts which is situated in altitudinal between 1300 to 1835 $\mathrm{m}$ above sea level. It's situated $50 \mathrm{~km}$ north of Asella, the zonal capital city and $25 \mathrm{~km}$ south of Adama town which is the most popular with MICE (Meeting, Incentive, conference and Exhibition) tourism. This makes the block to be visited all over the year by tourist. Even during wet season there is no mud to travel with vehicle and on foot.

2.1.2. Flora and fauna: The park features are Acacia woodland, bush land and scrubland; which are crucial components of the park ecosystem, keeping the highly fragile soil of the area from erosion (Dereje Mokonin, 2006). The major attractive fauna is bird species. Additionally, Mammal species like; Warthog, Greater Kudu, lesser kudu and Hyenas are common animals.

2.1.3. Socioeconomic: The people who settled in and around the park are the ethnic group of Oromo, Arsi clan. Farming practices is mainly dominating the surrounding areas of Dhera Dilfekar block (Dereje Mokonin, 2006). The major socio-economic problems are highly increasing number of human and livestock population density which could account for the boundary range shrinkages of Dhera Dilfekar bock. The bock is unsuitable for agronomy and heavy grazing but the local people use it for these purposes (DWAO, 2007).

\subsection{Methods of data collection}

This research paper is written based on both primary and secondary sources. To gather the primary data, semistructured questionnaires and field assessment were made to determine the opportunities for the development of ecotourism in Dhera Dilfekar block. The qualitative data were acquired through semi-structured questionnaire and interviews from selected groups. The researcher used purposeful sampling method to select informants. The interview was made with twenty-one (21) knowledgeable informants who were selected purposefully. Three (3) experts from the park, park warden, two (2) experts from culture and tourism office of Dodota district, and ten (10) elders from the local area. The sample size was determined based on the quality of information obtained from the informants. The secondary data were collected from journals, books, web sites and official documents. Also, inventory assessments were taken to collect the status of biodiversity resource through plant specimen collection, transect walk and field observation. An inventory was made using direct observation and specimen collection which is supported with field materials such as Nikon D3200 Digital camera, Nikon action 10x50 
Binocular, Garmin 60 GPS and materials for field plant press.

\subsection{Data Analysis}

The collected quantitative data were analyzed using descriptive and explanatory statistics; Microsoft Excel and SPSS (Statistical Package for Social Science) Version 25 software were used for the analysis. Qualitative data analysis followed steps of data reduction (selecting, focusing, simplifying, abstracting, transforming), data display (organizing, compressing), and conclusion drawing (Miles and Huberman, 1994). The findings were discussed through narrative and explanatory approaches then presented by using tables, figures, graphs, and charts.

\section{RESULTS AND DISCUSSION}

Assessment on ecotourism potentials at Dhera Dilfekar block was made based on natural attractions, cultural and historical features; from the perspective of identifying conservation challenges hindering the management activity and to indorse possible mitigation strategies.

\subsection{Ecotourism Potentials of Dhera dilfekar block}

The dhera dilfekar block is home for a variety of mammals and bird species. A common mammal species inhabited in this conservation block are Greater Kudu, Lesser Kudu, Spotted Hyena, Warthog, Anubis Baboon and others as well as a variety of bird species like Cattle Egret, White Browed Sparrow, Ring Necked Dove, Ruppels Starling and others. The vegetation cover was dominated by different acacia species like Acacia albida, Acacia tortilis, Acacia Senegal and Balenites aegyptica.

Additionally, the park harbors seven (7) major tourist attraction sites namely; Dire kiltu hyena valley, Dire kiltu lesser kudu site, Dire kiltu greater kudu site, Fachiso greater kudu site, Gara dima hyena valley(1), Awash bishola dhagaa taa'aa valley and Gara dima hyena valley(2) (Table 3.2. Circulation within the Park

3.2.1. Main gate /entrance/of Dhera Dilfekar block

There are two park gates(entrance) for dhera dilfekar block ; through dilfekar \& through Asphalt road from Adama to dhera Asella before you entered to the center of dhera town you will find park gate. The main gate of Dhera Dilfekar block was found on the main asphalt road from Adama to Asella. On the main gate there is tourist information center. When you entered to the park boundary you meet a guy inside tourist information center on the gate. Once you come to site at any time you have a great chance to see wild animal's especially large group of greater kudus.

On the other side near Dilfekar spiritual place, there is also another gate especially for tourists these back from Bale Mountains through Asella town. They can enter through this gate and can exit with the main gate that found on the asphalt road to the west direction from Adama to Asella main road.

There is car road and foot path which takes you to all direction of the park (to the east, west, north and south). Trekking is also possible on mount Dilfekar and Gara dima. The one who come with bicycle can also ride everywhere.

\subsubsection{Natural attraction of this block}

The major Core points of wildlife area were the following.
$\checkmark$ Dire kiltu hyena valley
Garadima hyena valley(1)
$\checkmark \quad$ Dire kiltu lesser kudu site
Garadima hyena valley (2)
$\checkmark \quad$ Dire kiltu greater kudu site Awash bishola Dhagaataa'aa

$\checkmark$ Fachiso greater kudu site 


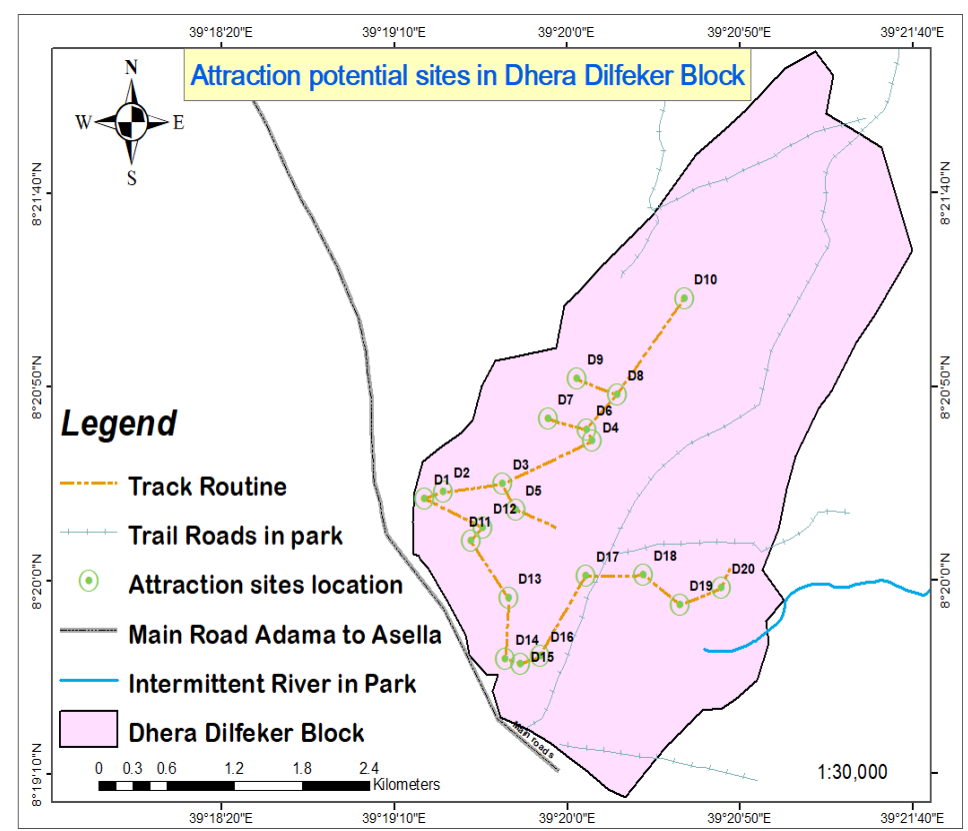

Figure 2.Dhera Dilfekar Block Tourism Attraction Potential Map

Table 1. Geographical Location of Attraction Places on the Block

\begin{tabular}{|c|l|c|c|}
\hline $\begin{array}{c}\text { Map } \\
\text { code }\end{array}$ & Name of Attraction places & Longitude & Latitude \\
\hline D1 & Main gate1(tourist information center) & 535436 & 921725 \\
\hline D2 & Town Area Out post & 535605 & 921780 \\
\hline D3 & Fachiso Water pond & 536129 & 921843 \\
\hline D4 & Gara Dima Hyena Valley Water Pond & 536925 & 922183 \\
\hline D5 & Ujune Baji Graveyard & 536244 & 921633 \\
\hline D6 & Gara Dima Hyena Valley1 & 536874 & 922267 \\
\hline D7 & Fachiso Greater kudu site & 536532 & 922360 \\
\hline D8 & Gara Dima (out post) & 537147 & 922552 \\
\hline D9 & Gara Dima Hyena Valley2 & 536789 & 922678 \\
\hline D10 & Awash Bishola dhagaa taha valley & 537739 & 923312 \\
\hline D11 & Town Area Water Pond & 535852 & 921388 \\
\hline D12 & Water Pond2 & 535954 & 921492 \\
\hline D13 & Mount Dilfekar & 536181 & 920939 \\
\hline D14 & Dilfekar spiritual place & 536154 & 920451 \\
\hline D15 & Main gate2 (Dilfekar) & 536290 & 920414 \\
\hline D16 & Mount Dilfekar Out post & 536462 & 920480 \\
\hline D17 & Dilfekar Water pond & 536867 & 921110 \\
\hline D18 & Dire Kiltu Greater Kudu Site & 537376 & 921118 \\
\hline D19 & Dire Kiltu Peak Point & 537699 & 920880 \\
\hline D20 & Dire Kiltu Lesser Kudu Site (out post) & 538067 & 921013 \\
\hline
\end{tabular}


Table 2.Major Tourist Attraction Sites within Arsi Mountains NP Dhera Dilfekar Block

\begin{tabular}{|c|c|c|c|c|}
\hline Map code & $\begin{array}{l}\text { Name of } \\
\text { Attraction }\end{array}$ & $\begin{array}{l}\text { Distance } \\
\text { from main } \\
\text { gate }(\mathbf{k m})\end{array}$ & What to See & What to do \\
\hline D1 & Main gate 1 & 0.00 & Tourist Information center & Pay entrance fee \\
\hline $\mathrm{D} 2$ & $\begin{array}{l}\text { Town Area Out } \\
\text { post }\end{array}$ & 0.17 & Overview of the landscape & $\begin{array}{l}\text { Game viewing, } \\
\text { photography, walking }\end{array}$ \\
\hline D3 & Fachiso water pond & 0.70 & $\begin{array}{l}\text { Wildlife drinking water } \\
\text { point, greater kudu, lesser } \\
\text { kudu, warthog }\end{array}$ & $\begin{array}{l}\text { Game viewing, } \\
\text { photography, walking }\end{array}$ \\
\hline$\overline{\mathrm{D} 4}$ & $\begin{array}{l}\text { Gara Dima Hyena } \\
\text { Valley Water Pond }\end{array}$ & 1.57 & $\begin{array}{l}\text { Wildlife drinking water } \\
\text { point, greater kudu, lesser } \\
\text { kudu, warthog }\end{array}$ & $\begin{array}{l}\text { Game viewing, } \\
\text { photography, walking }\end{array}$ \\
\hline$\overline{\mathrm{D} 5}$ & $\begin{array}{l}\text { Ujune Baji } \\
\text { Graveyard }\end{array}$ & 0.95 & Different graveyards & $\begin{array}{l}\text { Game viewing, } \\
\text { photography, walking }\end{array}$ \\
\hline D6 & $\begin{array}{l}\text { Gara Dima Hyena } \\
\text { Valley1 }\end{array}$ & 1.66 & Herd of Hyenas & $\begin{array}{l}\text { Game viewing, } \\
\text { Photography, Hiking }\end{array}$ \\
\hline D7 & $\begin{array}{l}\text { Fachiso Greater } \\
\text { kudu site }\end{array}$ & 2 & $\begin{array}{l}\text { Greater kudu, lesser kudu, } \\
\text { Warthog }\end{array}$ & $\begin{array}{l}\text { Game viewing, } \\
\text { photography, walking }\end{array}$ \\
\hline D8 & Gara dima peak & 2 & $\begin{array}{l}\text { Greater kudu, Hyena, } \\
\text { lesser kudu, Warthog }\end{array}$ & $\begin{array}{l}\text { Game viewing, } \\
\text { Photography, Hiking }\end{array}$ \\
\hline D9 & $\begin{array}{l}\text { Gara Dima Hyena } \\
\text { Valley2 }\end{array}$ & 2.44 & Hyenas, Greater Kudu & $\begin{array}{l}\text { Game Viewing, } \\
\text { Photography, Hiking }\end{array}$ \\
\hline D10 & $\begin{array}{l}\text { Awash Bishola } \\
\text { dhagaa ta'aa valley }\end{array}$ & 3 & $\begin{array}{l}\text { Klipspringer, Greater } \\
\text { Kudu, }\end{array}$ & $\begin{array}{l}\text { Game Viewing, } \\
\text { Photography, Hiking }\end{array}$ \\
\hline D11 & $\begin{array}{l}\text { Town Area Water } \\
\text { Pond }\end{array}$ & 0.71 & $\begin{array}{l}\text { Wildlife drinking water } \\
\text { point, greater kudu, lesser } \\
\text { kudu, warthog }\end{array}$ & $\begin{array}{l}\text { Game viewing, } \\
\text { photography, walking }\end{array}$ \\
\hline D12 & Water Pond 2 & 0.56 & $\begin{array}{l}\text { Wildlife drinking water } \\
\text { point, greater kudu, lesser } \\
\text { kudu, warthog }\end{array}$ & $\begin{array}{l}\text { Game viewing, } \\
\text { photography, walking }\end{array}$ \\
\hline D13 & Mount Dilfekar & 1.27 & $\begin{array}{l}\text { Greater kudu, lesser kudu, } \\
\text { Warthog, }\end{array}$ & $\begin{array}{l}\text { Viewing the spectacular } \\
\text { landscape, Dhera town, } \\
\text { Wanji factory sugarcane } \\
\text { plantation }\end{array}$ \\
\hline D14 & $\begin{array}{l}\text { Dilfekar spiritual } \\
\text { place }\end{array}$ & 1.75 & $\begin{array}{l}\text { Spiritual mosque, } \\
\text { entombment of local } \\
\text { people with different } \\
\text { artistic design }\end{array}$ & $\begin{array}{l}\text { Like Dire sheik Hussen } \\
\text { "muda" was undertaken two } \\
\text { times per a year }\end{array}$ \\
\hline D15 & $\begin{array}{l}\text { Main gate2 } \\
\text { (Dilfekar) }\end{array}$ & 2 & Overview of the landscape & $\begin{array}{l}\text { Game viewing, } \\
\text { photography, walking }\end{array}$ \\
\hline D16 & $\begin{array}{l}\text { Mount Dilfekar Out } \\
\text { post }\end{array}$ & 2.1 & $\begin{array}{l}\text { Greater kudu, lesser kudu, } \\
\text { Warthog }\end{array}$ & $\begin{array}{l}\text { Game viewing, } \\
\text { photography, walking }\end{array}$ \\
\hline D17 & Dilfekar water pond & 1.5 & $\begin{array}{l}\text { Wildlife drinking water } \\
\text { point, greater kudu, lesser } \\
\text { kudu, warthog }\end{array}$ & $\begin{array}{l}\text { Game viewing, } \\
\text { photography, walking }\end{array}$ \\
\hline D18 & $\begin{array}{l}\text { Dire Kiltu Greater } \\
\text { Kudu Site }\end{array}$ & 3.34 & $\begin{array}{l}\text { Greater kudu, Hyena, } \\
\text { lesser kudu, Warthog }\end{array}$ & $\begin{array}{l}\text { Game viewing, } \\
\text { photography, walking }\end{array}$ \\
\hline D19 & $\begin{array}{lll}\begin{array}{l}\text { Dire } \\
\text { Point }\end{array} & \text { Kiltu } & \text { Peak } \\
\end{array}$ & 3.74 & $\begin{array}{l}\text { Greater kudu, Hyena, } \\
\text { lesser kudu, Warthog }\end{array}$ & $\begin{array}{l}\text { Game viewing, village } \\
\text { viewing, photography }\end{array}$ \\
\hline $\mathrm{D} 20$ & $\begin{array}{l}\text { Dire Kiltu Lesser } \\
\text { Kudu Site }\end{array}$ & 4.13 & $\begin{array}{l}\text { Lesser kudu ,Hyena, } \\
\text { Greater kudu }\end{array}$ & $\begin{array}{l}\text { Game viewing, } \\
\text { photography, walking }\end{array}$ \\
\hline
\end{tabular}

\subsubsection{Hydrological features}

Since there is no natural river or spring water in dhera dilfekar block, six (6) water ponds were artificially constructed in the park at different places for the purpose of wildlife drinking water storage .Before this, most of the time wild animals move to find water outside the park boundary especially on asphalt road; at that time they were suddenly exposed to car accident but now due to this ponds these problems were partially solved. 


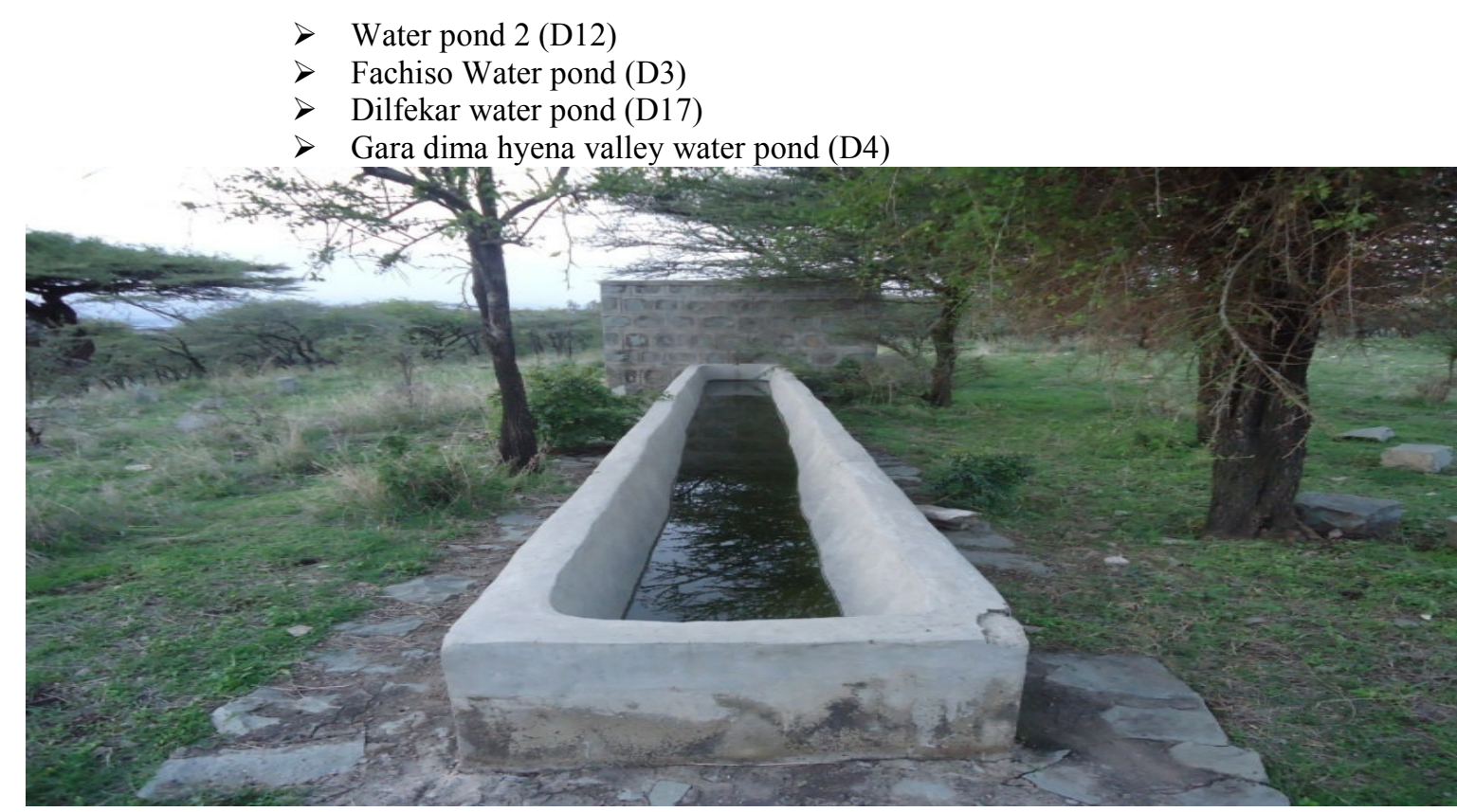

\subsubsection{Biological Resources}

Figure 3.Water Pond for Wildlife Drinking Water(Photo by: Ziyad J.)

\subsubsection{Wild Animals:}

The Park is home for about 25 wild mammals dominated by Greater kudu, lesser kudu, spotted hyena and Warthog. The other mammals such as Golden backed jackal, Abyssinian hare, Leopard, Grivet monkey, Anubis baboon, Grey duiker, etc are also found in this block(Abdurrahman Kubssa, 1995). According to the report of the park warden and tourist information center this block is the only place where visitors could watch a herd of greater and lesser kudu in the country without spending a minutes.

Table 3.The Wildlife Species found in Dhera Dilfekar Block

\begin{tabular}{|l|l|l|l|}
\hline No & Local Name & Common Name & Scientific Name \\
\hline $\mathbf{1}$ & Waraabessa & Spotted hyena & Crocuta crocuta \\
\hline $\mathbf{2}$ & Kuruphee & Grey duiker & Sylvicapra grimmia \\
\hline $\mathbf{3}$ & Gicii & Klipspringer & Oreotragus oreotragus \\
\hline $\mathbf{4}$ & Goljaa & Warthog & Phacochoerus aethiopicus \\
\hline $\mathbf{5}$ & Qeerransa & Leopard & Panther pardus \\
\hline $\mathbf{6}$ & Jeedala gurra bal,aa & Bat eared fox & Otocyton megalotis \\
\hline $\mathbf{7}$ & Deeroo & Serval cat & Felis serval \\
\hline $\mathbf{8}$ & Jeedala bakka maraa & Common jackal & Canis aureus \\
\hline $\mathbf{9}$ & Jaldeessa & Anubis baboon & Papio Anubis \\
\hline $\mathbf{1 0}$ & Qamalee & Monkey/vervet & Cercophitecus pygerythrus \\
\hline $\mathbf{1 1}$ & Awwaal-diigessa & Aardvark & Orycteropus afer \\
\hline $\mathbf{1 2}$ & Waraabessa ji'aa & Aard wolf & Protelus cristatus \\
\hline $\mathbf{1 3}$ & Dhaddee & Porcupine & Hystrix cristata \\
\hline $\mathbf{1 4}$ & Amaa & Mongoose/Egyptian & Herpestesin chneumon \\
\hline $\mathbf{1 5}$ & Hilleettii & Abyssinian Hare & Lepus capensis \\
\hline $\mathbf{1 6}$ & Amaa gaaguraa & Honey badger & Mellivora capensis \\
\hline $\mathbf{1 7}$ & Amaa gootaa/eegeeadii & White tailed mongoose & Icneumia albicauda \\
\hline $\mathbf{1 8}$ & Gadamsa gammojjii guddaa & Greater kudu & Tragelaphus strepsiceros \\
\hline $\mathbf{1 9}$ & Hammarreesa & Lesser kudu & Tragelaphus imberbis \\
\hline $\mathbf{2 0}$ & Waraabessa sarara qabu & Striped hyena & Hyena hyena \\
\hline $\mathbf{2 1}$ & Sardiida dugda gurrraacha & Black-backed jackal & Canis mesomelas \\
\hline $\mathbf{2 2}$ & Moor'ee & African civet & Civettictis cuivetta \\
\hline $\mathbf{2 3}$ & Warbaa/daalga anbassaa & Caracal & Felis caracal \\
\hline $\mathbf{2 4}$ & Deeroo & Serval cat & Leptailurus serval \\
\hline $\mathbf{2 5}$ & Amaa & Ichneumon mongoose & Herpestes ichneumon \\
\hline $\mathbf{2 6}$ & Jeedala gurra bal'aa & Bat eared fox & Otocyton megalotis \\
\hline
\end{tabular}



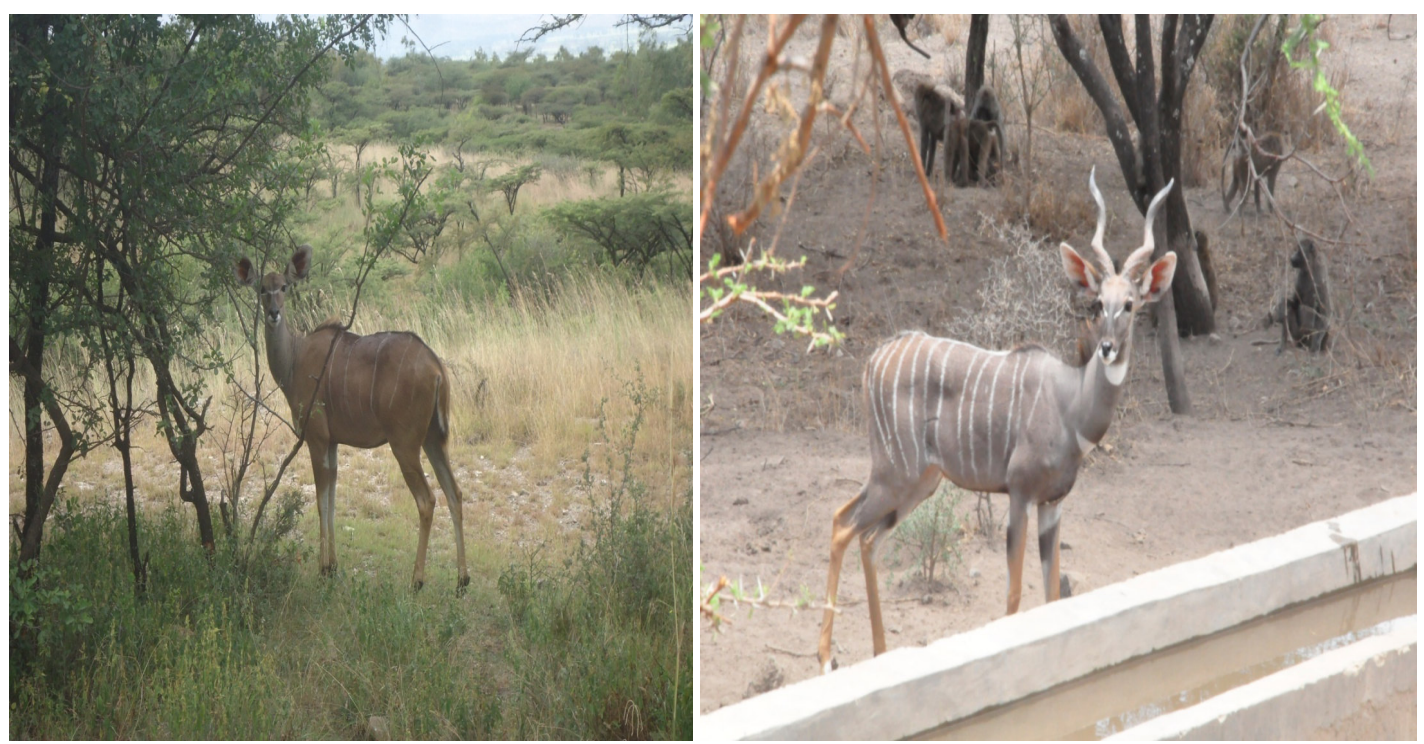

Figure 4. Greater Kudu (Left side) and Lesser Kudu (Right side(Photo by : Ziyad J.))

3.2.4.2. Vegetation cover: Dhera-Dilfeker has a variety of acacia dominant vegetation types like Acacia melifera, Acacia senegal, Acacia tortilis, Acacia albida , Acacia abyssinica, , Acacia seyal, Balenites aegyptica (Abdurrahman Kubssa, 1995).

Table 4.The common Plant Species found dhera dilfekar block

\begin{tabular}{|c|c|c|c|c|}
\hline S.no & $\begin{array}{l}\text { Local name } \\
\text { /Afan oromo/ }\end{array}$ & Scientific name & Family & Plant habit \\
\hline 1 & Doddotii & Acacia etbaica & Fabaceae & Shrub \\
\hline 2 & Qordiimoo & Acacia nilotica & Fabaceae & Tree \\
\hline 3 & Ajoo & Acacia oerfota & Fabaceae & Shrub \\
\hline 4 & Saphansa & Acacia senegal & Fabaceae & Tree \\
\hline 5 & Waaccuu & Acacia seyal & Fabaceae & Tree \\
\hline 6 & Loxxobbaa & Acacia tortilis & Fabaceae & Tree \\
\hline 7 & Qaraaruu & Acokanthera schimperi & Apocynaceae & Tree \\
\hline 8 & Shaartii & Asparagus africanus & Asparagaceae & Shrub \\
\hline 9 & Beddano & Balanites aegyptiaca & Balanitaceae & Tree \\
\hline 10 & Heebsata & Cadaba farinosa Forssk & Capparidaceae & Shrub \\
\hline 11 & Yeferenj Kitkita & Caesalipinia dectaptela & Fabaceae & Shrub \\
\hline 12 & Ceekataa & Calpurnia aurea & Fabaceae & Shrub \\
\hline 13 & Harangamaa & Capparis fascicularis & Capparidaceae & Shrub \\
\hline 14 & Agamsa & Carissa edulis & Apocynaceae & Shrub \\
\hline 15 & Shashawwee & Casuarina equisetifolia & Casuarinaceae & Tree \\
\hline 16 & Rukeessa & Combretum molle & Combretaceae & Tree \\
\hline 17 & Chal Gulfa & Cenchrus pennisetiformis & Poaceae & Herb \\
\hline 18 & Hammeessa & Commiphora africana & Burseraceae & Tree \\
\hline 19 & Gilay & Commiphora rostrata & Burseraceae & Shrub \\
\hline 20 & Wodeessa & Cordia africana & Boraginaceae & Tree \\
\hline 21 & Madheeraa & Cordial ovalus & Boraginaceae & Tree \\
\hline 22 & Bakkanniisaa & Croton macrostachus & Euphorbiaceae & Tree \\
\hline 23 & Baaladii /Magoof & Croton dichogamus & Euphorbiaceae & Tree \\
\hline 24 & Coqorsa & Cynodon dactylon & Poaceae & Herb \\
\hline 25 & Jirimee & Dichrostachys cinerea & Fabaceae & Shrub \\
\hline 26 & Dhittacha & Dodonea angustifolia & Sapindaceae & Shrub \\
\hline 27 & Mi'eessaa & Eculea schimperi & Ebenaceae & Shrub \\
\hline 28 & Ulaagaa & Ehertia cymosa & Boraginaceae & Tree \\
\hline 29 & Waleensuu & Erythrina abyssinica & Fabaceace & Tree \\
\hline 30 & Baargamoo & Eucalyptus camaldulensis & Myrtaceae & Tree \\
\hline 31 & Hadaamii & Euphorbia candelabrum & Euphorbiaceae & Shrub \\
\hline 32 & Qacaacillee & Fleuggea virosa & Euphorbiaceae & Shrub \\
\hline
\end{tabular}




\begin{tabular}{|l|l|l|l|l|}
\hline S.no & $\begin{array}{l}\text { Local name } \\
\text { /Afan oromo/ }\end{array}$ & Scientific name & Family & Plant habit \\
\hline 33 & Harooressa & Grewia bicolor & Tiliaceae & Tree \\
\hline 34 & Harooressa arbaa & Grewia mollis & Tiliaceae & Tree \\
\hline 35 & Qococcee & Grewia tenax & Tiliaceae & Shrub \\
\hline 36 & Sanbaleexa & Hyparrhenia multiplex & Poaceae & Herb \\
\hline 37 & Ashaalee & Leucas abyssinica & Lamiaceae & Shrub \\
\hline 38 & Qanqalcha & Maerua angolensis & Capparidaceae & Shrub \\
\hline 39 & Kombolcha & Maytenus senegalensis & Celastraceae & Shrub \\
\hline 40 & Qacama & Myrsine africana & Myrsinaceae & Tree \\
\hline 41 & Ejersa & Olea africana & Oleaceae & Tree \\
\hline 42 & Abushuukii & Opuntiaficus-indica & Cactaceae & Shrub \\
\hline 43 & Damakasee & Ocimium lamiifolium & Lamiaceae & Shrub \\
\hline 44 & Waatoo & Osyris quadripartita & Santalaceae & Shrub \\
\hline 45 & Biiqqaa & Pappea capensis & Sapindaceae & Tree \\
\hline 46 & Urgeessaa & Premna resinosa & Lamiaceae & Shrub \\
\hline 47 & Qonxiir & Pterolobium stellatum & Fabaceae & Tree \\
\hline 48 & Hawaasee & Rhus retinorrhoea & Anacardiaceae & Tree \\
\hline 49 & Xaaxessaa/mi,eessaa & Rhus natalensis & Anacardiaceae & Tree \\
\hline 50 & Hiddiibidoo & Solanum somalensis & Solanaceae & Shrub \\
\hline 51 & Hiddii & Solanum incanum & Solanaceae & Shrub \\
\hline 52 & Birreessa & Terminalia brownii & Combretaceae & Tree \\
\hline 52 & Qurquraa & Ziziphus mucoranata & Rhamnaceae & Tree \\
\hline 3.42 & Bird Species &
\end{tabular}

\subsubsection{Bird Species}

There are more than 189 birds among these; Helmeted Guinea fowl, little bee eater, Black wood hoopoe, Grey wood pecker, Grey headed sparrow, Shinning sun bird, Rupp ells long tailed starling, Red checked cordon blue, Speckled mouse bird, Ring necked dove, Black kite and Long crested eagle are the common species (Table 5).

Table 5. The Common Bird Species Found In Dhera Dilfekar Block

\begin{tabular}{|l|l|l|}
\hline No. & Common name & Scientific name \\
\hline 1 & Little bee eater & Merops pusillus \\
\hline 2 & African Hoopoe & Upupae pops \\
\hline 3 & Black wood hoopoe & Phoeniculus aterrimus \\
\hline 4 & Grey woodpecker & Dendropicos goertae \\
\hline 5 & Grey headed sparrow & Passer griseus \\
\hline 6 & Shinning sun bird & Nectarinia habessinica \\
\hline 7 & Ruppell's long tailed starling & Lamprototrispur puropterus \\
\hline 8 & Red checked cordon blue & Uraeginthus bengalus \\
\hline 9 & Speckled mouse bird & Colius striatus \\
\hline 10 & Ring necked dove & Strreptopelia capicola \\
\hline 11 & Laughing dove & Streptopelia senegalensis \\
\hline 12 & Helmented guinea fowl & Numida meleagris \\
\hline 13 & African hawk eagle & Hiera aetusspilogaster \\
\hline 14 & Black kite & Milvus migrans \\
\hline 15 & Superb starling & Spreo superbus \\
\hline 16 & Black headed batis & Batis minor \\
\hline 17 & African paradise monarchy & Terpsiphone viridis \\
\hline 18 & Speckled pigeon & Columba guinea \\
\hline 19 & Pied crow & Corvus albus \\
\hline 20 & African rock & Corvus capensis \\
\hline 21 & Tawny flanked prinia & Prinia subflava \\
\hline 22 & Hemprich's hornbill & Tockus hemprichii \\
\hline 23 & Sinnamon breasted rock bunting & Emberizata hapisi \\
\hline 24 & Village indigobird & Vidua chalybeate \\
\hline 25 & Red billed firefinch & Lagonosticta senegala \\
\hline 26 & Northern black tit & Parusleu comelus \\
\hline 27 & Blue breasted bee eater & Meropsvari egatus \\
\hline 28 & Stout cisticola & Cisticola robusta \\
\hline & & \\
\hline
\end{tabular}




\begin{tabular}{|l|l|l|}
\hline No. & Common name & Scientific name \\
\hline 29 & Northern red bishop & Euplectes franciscanus \\
\hline 30 & Morning weater & Oenanthe lugens \\
\hline 31 & Common bulbul & Pycnonotus barbatus \\
\hline 32 & Abyssinian crimson wing & Cryptospiza salvadori \\
\hline 33 & Eurasian redstart & Phoenicurus phoenicurus \\
\hline 34 & House bunting & Emberiza striolata \\
\hline 35 & Coppery sunbird & Cinnyris cupreus \\
\hline 36 & Red billed ox pecker & Buphagusery throrhynchus \\
\hline
\end{tabular}

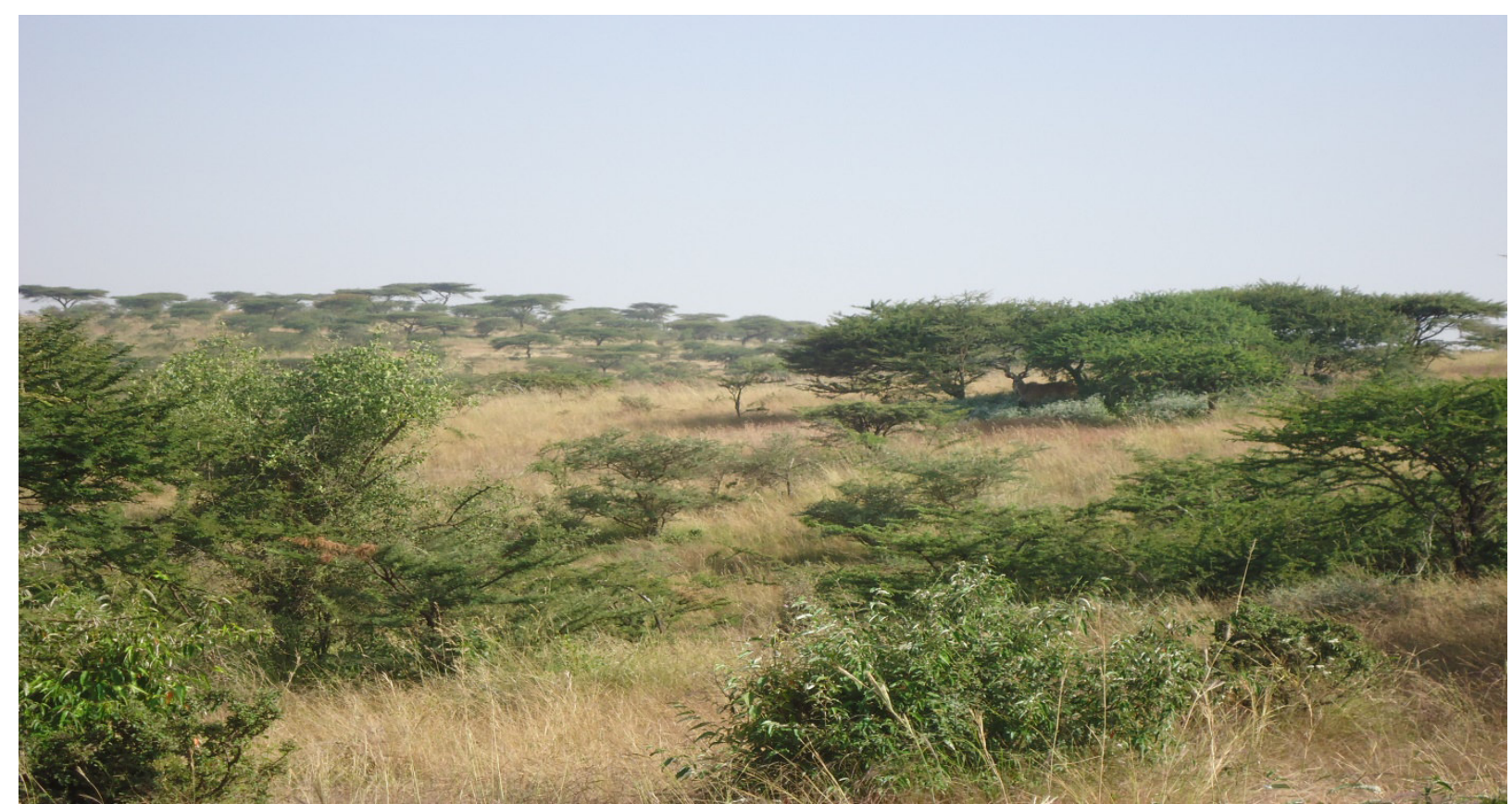

Figure 5.Vegetation cover of the dhera dilfekar block (Photo by: Ziyad J.)

\subsubsection{Historical and Cultural site}

In addition to natural attraction, there are also cultural and historical heritage sites where the local communities undertaking spiritual activities.

\subsubsection{Dilfekar Spiritual Place}

It is found at the South gate of Dhera block about $300 \mathrm{~m}$ far away from main road which goes to sire District to the north direction. It is a ritual/spiritual cultural site where "Muda" practiced and long aged with different size of entombment of local people with different artistic designed plate of wildlife habitat of the area. This burial may attract visitors because it has a beautiful artistic design with different type of picture. In this area like dire sheik Hussein muda was undertaken two times per a year (during Hajji and Zahra) hundreds of believers even come from faraway places for pilgrimage.

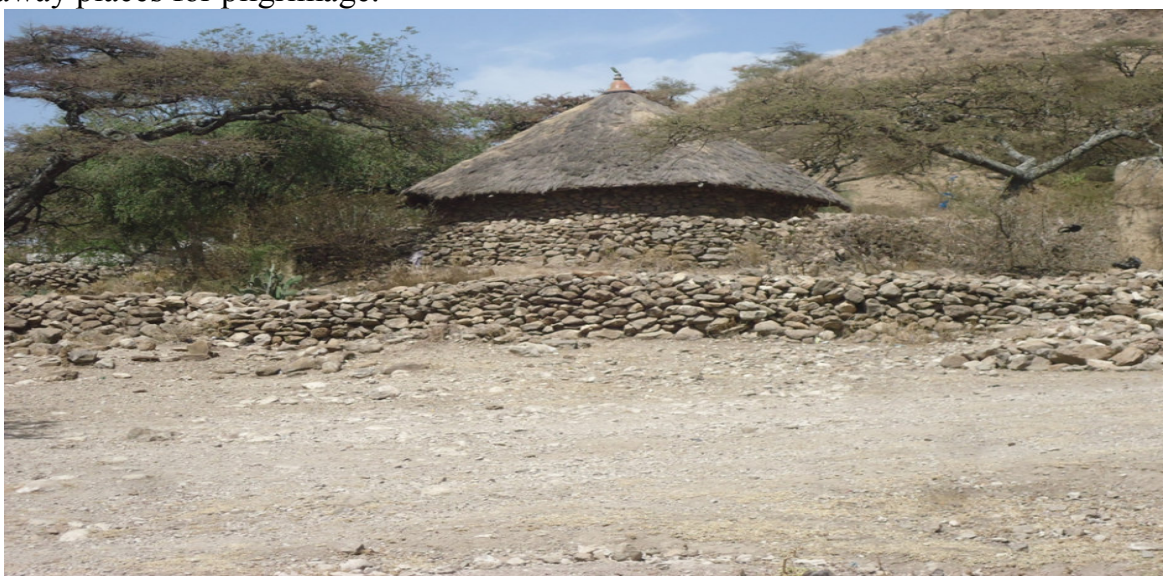

Figure 6.Dilfekar Spiritual Place(Photo by:Ziyad J.) 
3.2.5.2. Ujune Baji Graveyard and His Historical Background (Source: Local elders \&his relatives)

This grave was found in the dhera dilfekar block of Arsi mountains national park. It is about $1 \mathrm{~km}$ far from the first main gate. This grave may attract visitors because it has a beautiful artistic design with different type of animal pictures such as Ape, elephant and others showing the availability of diversity of wildlife in the area. In addition there are also cultural materials such as Shield, Spear for the purpose of defending his opponent.

Ujune Baji was a popular hero man that was born in Oromia regional state Arsi zone, dodota woreda ,dilfekar kebele. This person was very prominent and well known in Oromo Arsi area during war on Oromo people. During that time he tries to defend his enemies in collaboration with his brothers, Burka, Kilo and other friends. He has very strong horse called "biyyo" that he was used to ride for purpose of defending the war. He named his horse "'biyyo" 'because during that time there is no enough food to eat and famine was so known. So, to survive peoples eat soil which means in Afaan oromoo "biyyoo". He is patriotic person who always stand for truth, to protect his country from enemy. In addition, Ujune Baji was a person who always refuses to give his hand to his opponents and enemies. During that time according to local elders told us "Ujune Baji'"was a young person of 20 years old. He was born in 1867 E.C. He seriously struggles and won Menilik II war on oromo peoples especially in Arsi area. This person was a father of nine (9) children. He follows "wakeffata" religion. Finally he become sick and left this world during this war.

\subsubsection{The Local Community}

The people who settled around the park are ethnic group of Oromo-Arsi. They have their own unique dressing style (Figure 6), wedding ceremony, local handicrafts, traditional foods, and time-honored ruling system of Abba Gada. Some of the known traditional foods are called "Chechebsa" and" Chuko" with traditional coffee ceremony. They are welcoming and friendly people and endowed with traditional way of living.

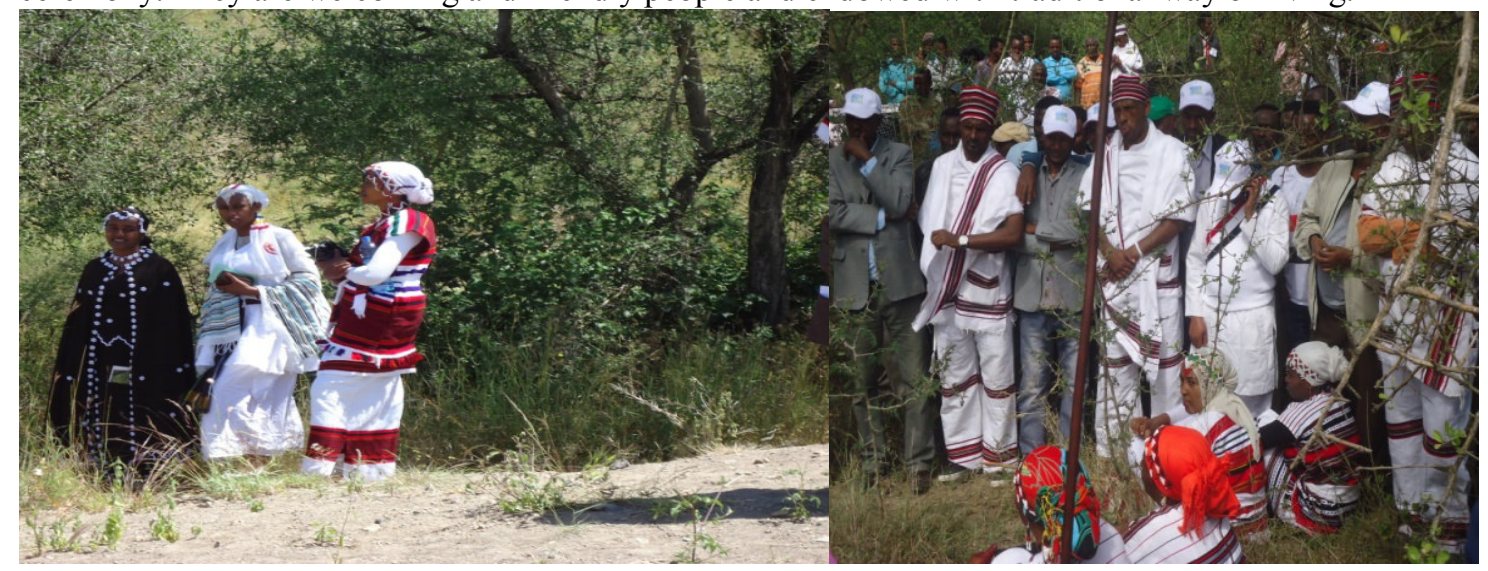

Figure 7. Arsi Oromo Cultural Dressing Style(Photo by:Ziyad J.)

\section{CONCLUSION AND RECOMMENDATIONS}

Eco tourism potential of Arsi mountains National Park dhera dilfekar block is assessed based on natural attractions, cultural features, and tourism activities. The results of the research revealed some of ecotourism potentials of dhera dilfekar block like Dire kiltu hyena valley, Dire kiltu lesser kudu site, Dire kiltu greater kudu site , Fachiso greater kudu site, Gara dima hyena valley(1), Awash bishola dhagaa taa'aa valley and Gara dima hyena valley (2) (Table 2). Additionally the park can offer the visitor an opportunity to see lowland game species, a variety of bird species, Acacia dominated spectacular landscape, historical and Cultural sites, Cultural living style of the local community which makes this block unique feature to attract eco tourists. Furthermore, tourist facilities and services including camping sites, guiding services and hotels/lodges nearby found in Adama town which is the center of conference tourism and sodare resort hotel which is frequently visited by both domestic and foreign tourists contributed to an increase in tourist flow.

Wild animals like Greater kudu, Lesser Kudu, Warthog, Hyena, Anubis baboon, are among common species in dhera dilfekar block. The park is also rich in Avifauna species (OFWE, 2010).

Recommendations: Based up on the above conclusions the following recommendations were made.

$\checkmark$ To scale up wildlife tourism potentials of dhera dilfekar block, better promotion with practical conservation activities, community based tourism approaches and infrastructures should be implemented for tourist destination of dhera dilfekar bock.

$\checkmark$ On the other hand, anthropogenic pressures are identified as the main conservation challenges; hence the park requires immediate intervention.

$\checkmark$ Awareness creation should be developed for the local communities and other stakeholders on the benefits of ecotourism and conservation of eco-attractions for sustainable management of ecotourism activities. 
$\checkmark \quad$ Finally it is better that offering tourist facilities and services and creating job opportunities for members of local communities have a positive impact on tourism activities by creating a sense of ownership on the local community.

\section{Acknowledgement}

We are grateful to Almighty Allah who helped us to accomplish this study. Our gratitude also goes to our families who encouraged and supported us in any aspect of our life and in the work of the present study.

\section{REFERENCES}

Abdurrahman Kubssa .1995. A survey in Dhera Dilfaqar Protected area .Oromia Natural Resources Development and Environmental protection Bureau, Finfinnee.

Alamneh Amare. 2015. Wildlife Resources of Ethiopia: Opportunities, Challenges and Future Directions: From Ecotourism Perspective: A Review Paper. Natural Resources, 6: 405-422 Pp.

Cooper C. \& Hall M. 2008. Contemporary Tourism: An International Approach London, ButterworthHeinemann.

Dereje Mekonnen. 2006. Woody Species Composition of Dilfaqar Regional Park and Its Socio-Economic Importance.

DWAO (Dodota Woreda Agricultural Office) .2007. Annual Report Report, 18 Pp.

Getachew Bantihun, Shimekit Tadele, \& Abebe Ameha. 2020. Avian Diversity in Dilifekar Block, Arsi Mountains National Park, Ethiopia. Advances in Bioscience and Bioengineering, 8(1): 6-11

King, D. \& Stewart, W.1996. Ecotourism and commodification: protecting people and places. Biodiversity \& Conservation, 5(3), 293-305 pp.

Mowforth, M. \& Munt I. 1998.Tourism and sustainability. New Tourism in the Third World. London and New York: Routledge.

Mustefa Sultan and Teyiba Amano .2017. Eco-Tourism Business Plan as Strategy on Income Generating Schemes from Non-Timber Forest Product (NTFPs) for Arsi Mountains National Park, Dhera-Dilfaqar block, Oromia, Ethiopia.

Oromia Forest and Wildlife Enterprise (OFWE). 2018. Arsi Mountains National Park Wildlife Monitoring Report.

Papatheodorou A. 2006. Managing Tourism Destinations, Northampton, Edward Elgar Publishers.

Sinha, C. 2001. Wildlife Tourism: A Geographical Perspective. Conference Paper, 1-23pp.

UNWTC .2010.World Guide for Local Authorities on Developing Sustainable Tourism. First Edition, WTO.

Ziyad Jemal \& Kedir Abdurahman. 2019. Wildlife Resources, Conservation Challenges and Management Strategies in Ethiopia: A Review Paper.1-4pp. 